\title{
CORRIGENDUM: \\ ON A NEW KIND OF EINSTEIN WARPED PRODUCT (POLJ)-MANIFOLD
}

\author{
A. PIGAZZINI, C. ÖZEL ${ }^{\dagger}$, P. LINKER AND S. JAFARI
}

Date of Receiving : 27. 03. 2020

The paper [1] requires some corrections in our calculations, in particular we were wrong to consider the skew symmetry of the Riemann tensor, so the equations (2.5) and (2.7) in [1] become:

$$
\left\{\begin{array}{l}
\Delta f-\frac{c}{m} f^{2}=0 \\
(m+1)|\nabla f|^{2}+\frac{c}{m} f^{3}=0
\end{array}\right.
$$

We wrote $(m+1)$ instead $(m-1)$ because the skew symmetry of the Riemann curvature tensor causes one the terms in the trace to vanish, and since $m$ must be negative, the terms is eliminated by adding 1 . In this scenario the $(2,-2)$-POLJ manifold does not exist, but exists the (3,-3)-POLJ manifold. In case where $n=3, m=-3$, $\lambda=\mu=0, R_{B}=R_{f_{B}}, h \neq 0$ and by setting $x=(2 / h) f$, the equations (2.8) and (2.9) in [1] become: (1) $\Delta x-2 x^{2}=0$ and (2) $|\nabla x|^{2}-x^{3}=0$. The technique used remains the same as [1] and by performing the new calculations we obtain: (3) $\omega_{2}^{2}+\omega_{3}^{2}=x^{(1 / 2)}\left(E d y^{2}+2 F d y d z+G d z^{2}\right)$, for some functions $E, F$ and $G$ on the domain of the coordinate charte $(x, y, z)$ that satisfy $E G-F^{2}=1$. Now assume that our metric has the form: (4) $g=x^{-3} d x^{2}+\left(x^{a} d y\right)^{2}+\left(x^{b} d z\right)^{2}$, then this is equivalent to having: (5) $2 a+2 b-1=0$ and the condition that $R_{f_{B}}+6 x=0$ (i.e. the $f$-curvature-Base [2]), since the scalar curvature is: $R_{B}=-\left[2\left(a^{2}+a b+b^{2}\right)+a+b\right] x$. Hence the equation becomes: (6) $6-2\left(a^{2}+a b+b^{2}\right)-a-b=0$. Since these two equations $((5)$ and $(6))$ are satisfied only for $a=1, b=-1 / 2$, or $a=-1 / 2, b=1$, then we have the following metric: $g=x^{-3} d x^{2}+x^{2} d y^{2}+x^{-1} d z^{2}$. Then our $(3,-3)$-POLJ manifold metric is: $d s^{2}=x^{-3} d x^{2}+x^{2} d y^{2}+x^{-1} d z^{2}-\left((c x)^{2} / 36\right)\left(d \xi^{2}+d \varphi^{2}+d \varrho^{2}\right)_{-3}$. We underline that the fiber-manifold (derived-manifold with negative dimension) is intended as $\mathbb{R}^{d}+$ bundle of obstruction ([3]), therefore on $F$ we consider the valid Riemannian geometry defined on $\mathbb{R}^{d}$ paying attention to the dimension.

2010 Mathematics Subject Classification. 53C25.

Key words and phrases. Einstein warped product, derived-differentials, negative dimensional manifolds, invisible-manifolds, point-manifolds, derived-manifold.

Communicated by: Shiv.K.Kaushik

${ }^{\dagger}$ Corresponding author. 